\title{
Preparation and Control of the Formation of Single Core and Clustered Nanoparticles for Biomedical Applications Using a Versatile Amphiphilic Diblock Copolymer
}

\author{
Hongwei Chen ${ }^{1}$, Julie Yeh ${ }^{1}$, Liya Wang ${ }^{1}$, Hafsa Khurshid ${ }^{2}$, Nan Peng $^{1,3}$, Andrew Y. Wang ${ }^{4}$, and Hui Mao ${ }^{1}(\varangle)$ \\ ${ }^{1}$ Department of Radiology, Center for Systems Imaging, Emory University School of Medicine, Atlanta, Georgia 30322, USA \\ ${ }^{2}$ Department of Physics and Astronomy, University of Delaware, Newark, Delaware 19716, USA \\ ${ }^{3}$ Department of Rehabilitation Medicine, Chinese PLA General Hospital, 28 Fuxing Road, Beijing 100853, China \\ ${ }^{4}$ Ocean NanoTech, LLC, 2143 Worth Lane, Springdale, Arkansas 72764, USA
}

Received: 18 July 2010 / Revised: 18 August 2010 / Accepted: 9 October 2010

(C) The Author(s) 2010. This article is published with open access at Springerlink.com

\begin{abstract}
We report the application of a versatile diblock copolymer, poly(ethylene oxide)-b-poly( $\gamma$-methacryloxypropyl trimethoxysilane) (PEO- $b$-P $\gamma \mathrm{MPS}$ ), to prepare nanocrystals such as iron oxide nanoparticles or quantum dots, with either a single core or multi-core cluster, for biomedical applications. This amphiphilic copolymer comprises both a hydrophilic PEO segment and a hydrophobic segment with a "surface anchoring moiety" (the silane group) which can interact effectively with the hydrophobic nanocrystals through ligand exchange. One of the unique features of this work is that we can control the formation of either single core nanoparticles or multi-core nanoclusters by simply varying the conditions of ligand exchange and aging of the mixture of block copolymer and nanoparticles without needing to change the copolymer. The morphologies of the resulting single core nanoparticles or multi-core nanoclusters were confirmed by dynamic light scattering and transmission electron microscopy. The clustered nanoparticles exhibit enhanced physicochemical properties that are beyond those expected from a simple accumulation of individual nanoparticles. Additionally, the hybrid nanoparticles containing both magnetic iron oxide nanoparticles and optical quantum dots obtained using our strategy provide have combined magnetic and optical functionalities that allow for potential new and expanded biomedical applications, as demonstrated by their use for magnetic resonance imaging and biomarker-targeted cell imaging.
\end{abstract}

\section{KEYWORDS}

Magnetic nanoparticles, quantum dots, nanoparticle coating, copolymer, multifunctional nanoparticles, imaging

\section{Introduction}

Nanoparticles, such as magnetic iron oxide nanoparticles (IONPs) and semiconductor quantum dots (QDs), offer a range of unique and tunable physicochemical properties for developing biomedical applications [1], including magnetic resonance imaging (MRI) contrast agents in the case of IONPs [2] and ultra-bright and multiplex fluorescent labeling agents in the case of QDs [3]. However, most high

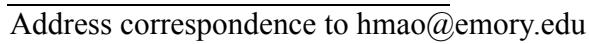


quality nanocrystals synthesized in the solid phase or in organic solvents and stabilized with hydrophobic ligands are not readily applicable for biological applications without appropriate surface modifications and functionalization to make them soluble in water without undergoing aggregation under physiological conditions [4].

Although some small molecule coating materials can be used to make nanocrystals water soluble $[5,6]$, biocompatible polymers play a very important role in functionalizing nanocrystals for biomedical applications, such as imaging and drug delivery $[7,8]$. These polymers can be divided into two categories. One class of polymers can be bound onto nanoparticles due to multivalency effects or ligand exchange [9-11] They are usually water soluble and can make nanocrystals monodisperse. Gao et al., for instance, developed an amphiphilic triblock copolymer which can be employed to coat nanoparticles via ligand exchange and make hydrophobic nanocrystals monodisperse in aqueous solution [9]. The other class comprises block copolymers which cannot undergo ligand exchange, but can self-assemble into micelles in water and encapsulate nanocrystals by the simultaneous co-precipitation method [12-16]. As well as single core nanoparticles, stabilized nanoparticle clusters can also be prepared and controlled using such block copolymers. In this case, the hydrophilic blocks form the corona, which provides the stabilization, while the hydrophobic blocks isolate the core of the nanoparticles from the solvent [17]. Ai et al. [12] studied poly( $\varepsilon$-caprolactone)-b-poly(ethylene glycol) (PCL- $b$-PEG) as a source of micelles to encapsulate multiple magnetic nanoparticles to form nanoparticle clusters. Kim et al. also investigated the self-assembly of multicomponent nanoparticles using the block copolymer polystyrene- $b$-poly(acrylic acid) (PS-b-PAA) [14]. Nanoparticles with different morphologies have different advantages in terms of biomedical applications. For single core nanoparticles, the minimum hydrodynamic size is favorable for in vivo applications [18]. The capabilities and functionalities of clustered nanoparticles may be enhanced compared with individual nanoparticles, leading to expanded biomedical applications $[12,19]$. It is worth noting that for the polymer coatings reported in the literature, different polymers are required to stabilize either single core or clusters of nanoparticles. To our knowledge, previously reported coating polymers are capable of forming and stabilizing either monodisperse single core or clustered nanocrystals, but are not versatile enough to do both.

Here we demonstrate that when our newly developed polysiloxane-containing amphiphilic diblock copolymer, poly(ethylene oxide)- $b$-poly( $\gamma$-methacryloxypropyl trimethoxysilane) (PEO- $b$-P $\gamma \mathrm{MPS}$ ) [20], is employed as a coating for hydrophobic nanocrystals, either single core nanocrystals or multi-core products of the same or different types of nanocrystals (e.g., IONPs and QDs) can be obtained by varying the coating conditions. The versatility and controllability of this block copolymer in forming either single or multi-core nanoparticles is likely due to the hydrophobic P $\gamma$ MPS block. By enhancing ligand exchange with nanocrystals through aging the copolymer and facilitating the "anchoring" of P $\gamma$ MPS blocks on the surface of nanocrystals [5, 11], water-soluble nanoparticles with a single core were generated. Conversely, minimizing the ligand exchange by not aging the mixture of copolymer and nanocrystals resulted in the formation of multi-core nanocrystal clusters, with a number of nanoparticles encapsulated during self-assembly of the block copolymers in water [12]. By assembling multiple nanocrystal cores, multi-core nanoparticles, e.g., IONP clusters, QD clusters or IONP-QD hybrids, can be obtained which exhibit magnetic and optical properties and/or multifunctional capabilities that can enhance and expand their biomedical applications, such as MRI contrast enhancing and biomarker targeted cell imaging.

\section{Experimental}

\subsection{Materials}

$\gamma$-Methacryl oxypropyltrimethoxysilane ( $\gamma$ MPS, 98\%, Aldrich) was purified by distillation under reduced pressure. 2,2-Azobis(isobutyronitrile) (AIBN, 98\%, Aldrich) used as an initiator was purified by recrystallization in ethanol. Sulfosuccinimidyl-4-( $N-$ maleimidomethyl), cyclohexane-1-carboxylate (SulfoSMCC, Pierce Biotechnology), and Cyclo(Arg-Gly- 
Asp-D-Phe-Cys) (RGD-SH, Peptides International, Inc) were used as received. Carbon disulfide (99.9\%), magnesium turnings (> 99.5\%), 2-chloro-2-phenylacetyl chloride (CPAC, 90\%), poly(ethylene oxide) monomethyl ether (PEO) $\left(M_{\mathrm{n}}=5000 \mathrm{~g} / \mathrm{mol}, M_{\mathrm{w}} / M_{\mathrm{n}}=1.10\right)$, anhydrous dioxane $(99.8 \%)$, anhydrous tetrahydrofuran (THF, 99.8\%), (3-aminopropyl)trimethoxysilane (APTMS), and all other chemicals were purchased from Aldrich.

\subsection{Synthesis and coating of nanoparticles}

Hydrophobic iron oxide nanocrystals were prepared by heating iron oxide powder and oleic acid in octadecene at $315^{\circ} \mathrm{C}$ [21]. CdSe/ZnS QD nanocystals with emission peaks at 576 or $604 \mathrm{~nm}$ were prepared using the typical successive ionic layer adsorption and reaction, or solution atomic layer epitaxy techniques [22]. PEO- $b$-P $\gamma$ MPS diblock copolymer was synthesized by reversible addition fragmentation chain transfer (RAFT) polymerization as previously reported [23]. The method for coating single core nanocrystals has been reported elsewhere [20]. For preparing nanoclusters of IONPs and QDs, the fresh mixture of nanocrystals and polymer solution without aging was added dropwise to water. For the preparation of IONP-QD hybrid nanoparticles, the same volume $(1.0 \mathrm{~mL})$ of IONPs $(5.0 \mathrm{mg} / \mathrm{mL}$ in THF) and QDs $(1.0 \mathrm{mg} / \mathrm{mL}$ in THF) which had been aged in the polymer coating solution were mixed together. The mixture was added dropwise to $10 \mathrm{~mL}$ of water. After dialysis with water, the resulting solution was purified four times by means of a magnet.

\subsection{Characterizations of nanoparticles}

The average hydrodynamic diameter and size distribution of the as-obtained single core nanoparticles and nanoclusters were measured using a dynamic light scattering (DLS) instrument (Malvern Zetasizer Nano S-90). The core sizes of the copolymer-coated nanocrystals and the thickness of the polymer layers were viewed by transmission electron microscopy (TEM, Hitachi H-7500 (75 kV)). Fluorescence absorption spectra were obtained with a scanning spectrophotometer (Shimadzu UV-2401PC). Photoluminescence spectra were acquired using a spectrofluorometer (SPEX FluoroMax-2). The saturation magnetization (Ms) value was measured with a vibrating sample magnetometer (Lakeshore, Inc.). The applied magnetic field was varied from 10,000 to $-10,000$ Oe. The $M s$ in emu/g Fe was normalized to the iron concentration which was determined by spectrophotometry [24]. MRI experiments were carried out on a 3 Tesla MRI scanner (Siemens Medical Solutions, Erlangen, Germany). For measurements of transverse relaxation time, $T_{2}$, which is a parameter measuring the MRI contrast effect, a multi-echo spin-echo sequence was used to simultaneously collect 20 data points at different echo times (TE $=6$ to $180 \mathrm{~ms}$ with an increment of $6 \mathrm{~ms})$ [12].

\subsection{Conjugation of cell targeting RGD ligands and cell binding assay}

Hybrid IONP-QD nanoparticles functionalized with amine groups were conjugated with the thiolated small peptide RGD-SH through a heterobifunctional linker Sulfo-SMCC [20]. For testing the binding of nanoparticles to cancer cells, U87MG human glioma cell line, which over express the tumor integrin $\alpha_{v} \beta_{3}$, was maintained in a humidified incubator (95\% air; $5 \% \mathrm{CO}_{2}$ ) at $37{ }^{\circ} \mathrm{C}$ [20]. Approximately $10^{5} \mathrm{U} 87 \mathrm{MG}$ human glioma cells were seeded in each cell culture chamber. Cells were fixed with $4 \%$ paraformaldehyde before the addition of targeted RGD-conjugated hybrid nanoparticles or non-targeted nanoparticles. Afterwards, $100 \mathrm{nmol} / \mathrm{L}$ RGD-conjugated IONP-QD hybrid nanoparticles or hybrid nanoparticles without RGD were added to two chamber slides, and the incubation was performed at room temperature for $2 \mathrm{~h}$ with gentle shaking. Subsequently, one slide was processed with Prussian blue staining for iron followed by nuclear fast red counterstaining for cells and then viewed by an optical microscope. The other slide was stained with 4',6-diamidino-2-phenylindole (DAPI) for cell nuclei and then mounted for examination using a confocal microscope (LSM 510, Carl Zeiss, Oberkochen, Germany). QDs were excited with a 543 $\mathrm{He}-\mathrm{Ne}$ laser and the images were captured with a $560-615 \mathrm{~nm}$ band pass filter. 


\section{Results and discussion}

By controlling the aging process of the mixture of copolymer PEO- $b$-P $\gamma$ MPS and hydrophobic IONPs (with a core size of $\sim 13 \mathrm{~nm}$ and coated with oleic acids) in THF, aqueous soluble monodisperse single core IONPs or multiple core clusters of IONPs were obtained after the nanocrystals were transferred into water, as shown schematically in Fig. 1(a). DLS (Fig. 1(b)) showed the formation of different species with overall hydrodynamic sizes of $23 \mathrm{~nm}$ and $58 \mathrm{~nm}$ for single core and clustered IONPs, respectively. Single core and clustered IONP solutions can both be stored at $4{ }^{\circ} \mathrm{C}$ for at least 6 months without any change in hydrodynamic size as monitored by DLS. TEM images (Figs. 1(c) and 1(d)) further confirmed the formation of both single core and clustered IONPs stabilized by the same copolymer. Monodisperse single core IONPs were composed of a visible thin polymer layer around each IONP as seen from TEM images. It is estimated that the thickness of the polymer layer is around 3-4 nm, based on TEM images. Since the IONP core diameter is $13 \mathrm{~nm}$, the total diameter of $19-21 \mathrm{~nm}$ measured from TEM is slightly lower than that measured by DLS. The higher value given by DLS may be attributed to the hydrodynamic effect in water. In contrast to the single core nanoparticles, IONP clusters formed from multiple cores were composed of an average of 8 single iron oxide nanocrystals and had an average size of $50 \mathrm{~nm}$ as measured by TEM. It is noticable that nanocrystals coated with our copolymer, especially the single core nanoparticles, tend to crowd together on the TEM grid, even though DLS data suggested that they were not aggregated. This may be attributed to the somewhat "fluidic" or less rigid nature of this copolymer and the short range isotropic van der Waals interactions and long range

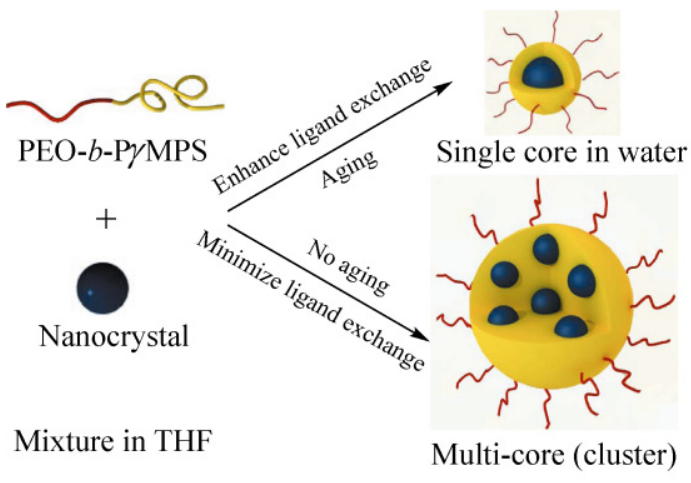

(a)

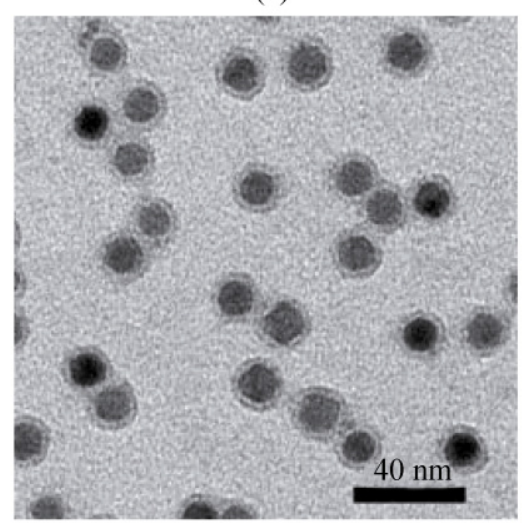

(c)

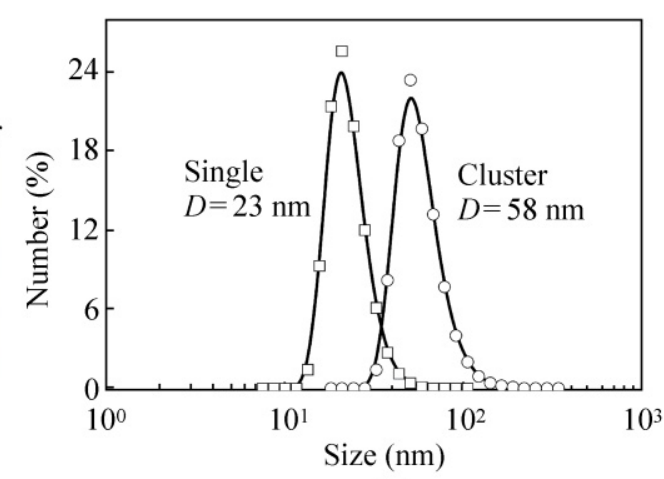

(b)

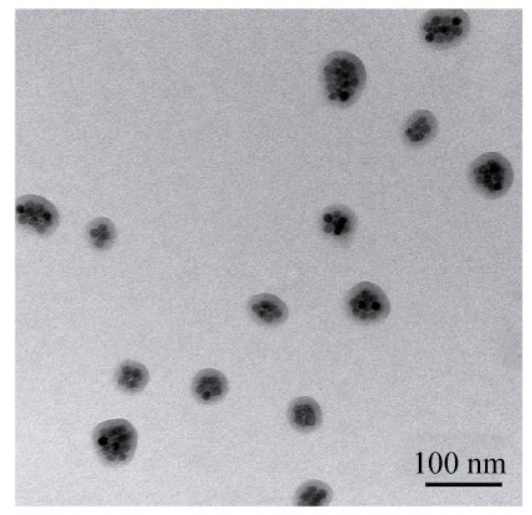

(d)

Figure 1 (a) Schematic illustration of the stabilization of nanoparticles with a single core or a multi-core in water using the amphiphilic diblock copolymer PEO- $b$-P $\gamma$ MPS. (b) The hydrodynamic size distributions of the single core and clustered nanoparticles in water. TEM images of PEO- $b$-P $\gamma$ MPS-coated single core (c) and clustered (d) IONPs 
anisotropic dipolar forces between the nanoparticles when deposited on a TEM grid, as first predicted by Luttinger and Tisza $[25,26]$. It is important to stress that both single core and clustered IONPs were prepared with the same coating polymer, which has not been previously reported, to the best of our knowledge.

Some recent reports have shown that the MRI contrast, e.g., the signal drop with $T_{2}$ weighted imaging, can be significantly enhanced by clustered IONPs [12, 27-30]. However, the samples of single or clustered IONPs employed were coated with different materials which may have affected their magnetic properties $[12,28,31]$. Our measurements of transverse $T_{2}$ relaxation times showed that the clustered IONPs exhibit an approximate 3.7-fold increase in the transverse relaxation rate $R_{2}\left(1 / T_{2}, \mathrm{~s}^{-1}\right)$ when compared to that of single core IONPs coated with the same copolymer, providing higher MRI contrast at the same Fe concentration (Fig. 2(a)). This significant enhancement in MRI contrast effect may be attributed to the synergistic magnetism of multiple IONPs as suggested by Lee et al. [32, 33]. In their study, similar results were observed using a core-satellite nanoparticle complex formed with multiple IONPs in a silica matrix [32]. In the amphiphilic copolymer-encapsulated nanoparticles, the hydrophobic blocks of the copolymer allow for isolation of nanocrystal cores. Each nanoparticle is discrete, resulting in the enhanced physicochemical properties of the nanoparticle clusters compared with those of the monodisperse single core nanoparticles $[12,34]$. It is worth noting that nanoparticles trapped in liposomal or phospholipid structures tend to form agglomerates and, therefore, they may not achieve the maximum physicochemical properties associated with individual nanoparticles acting collectively [35]. The enhancement of the physical properties of the clusters was also demonstrated by measurement of the saturation magnetization (Ms). Figure 2(b) shows the magnetization curves and hysteresis loops for both single core and clustered IONPs at room temperature. The hysteresis loops show almost zero coercivity for both monodisperse single core and clustered IONPs, indicating the superparamagnetic behavior of both materials. The Ms values for the single core and clustered IONPs are 41.7 and 110.3 $\mathrm{emu} / \mathrm{g} \mathrm{Fe}$, respectively. As expected, the IONP cluster has a much higher Ms than that of its single core counterpart with the same core size. Interestingly, the Ms of clustered IONPs is higher than that of $150 \mathrm{~nm}$ single dispersed magnetite nanoparticles $(75.6 \mathrm{emu} / \mathrm{g}$ ) reported by Goya et al. [36] as well as the theoretical value of bulk magnetite $(92.0 \mathrm{emu} / \mathrm{g}$ ) [37]. Since the $M s$ value has been normalized for the amount of iron, the higher magnetization of the IONP clusters reveals that the unique morphology of the clusters prepared with our amphiphilic copolymer may enhance the magnetic properties of IONP, which is not possible for a single particle counterpart. We believe that the

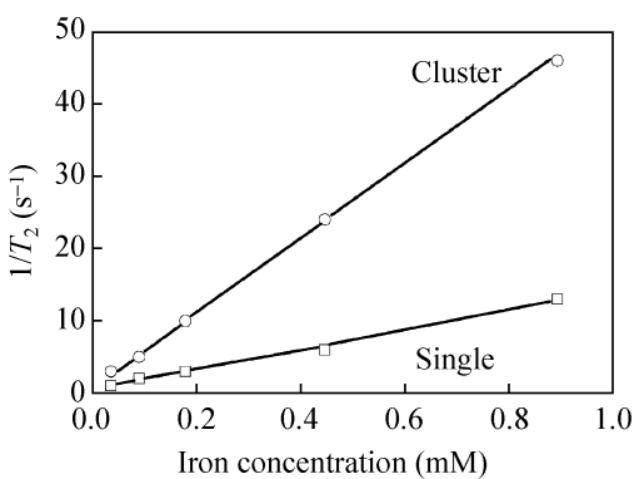

(a)

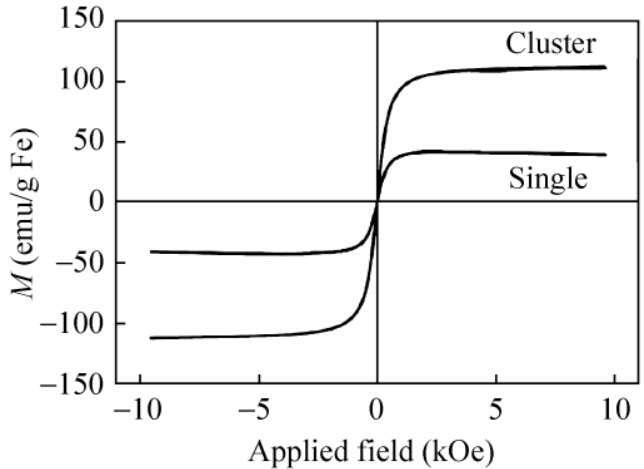

(b)

Figure 2 (a) Plots of the linear correlations of transverse relaxation rates $\left(1 / T_{2}, \mathrm{~s}^{-1}\right)$ of PEO- $b$-P $\gamma$ MPS-coated monodisperse single core $(\square)$ and clustered $(\circ)$ IONPs against the iron concentration $(\mathrm{mmol} / \mathrm{L})$, allowing the transverse relaxivities of each nanoparticle formation to be derived from the slopes of the plots. (b) Magnetization of monodisperse single core and clustered IONPs as a function of the applied magnetic field 
ultrahigh Ms value of the clustered IONPs may offer greater capabilities for biomedical applications, such as MRI contrast enhancement, hyperthermia therapy [38], and magnetic separations [39].

A simple strategy, similar to that for making monodisperse single core or clustered IONPs, was used to coat single core optical nanoparticle CdSe/ZnS QDs QDs or form clusters of QDs in aqueous solution. Figures 3(a) and 3(b) show TEM images of PEO- $b$-P $\gamma$ MPS copolymer-coated QDs in single core and cluster formations, respectively. The QD clusters consisted of an average of 12 QDs. DLS measurements of the hydrodynamic sizes of the two formations of QDs in solution showed overall sizes of $12 \mathrm{~nm}$ for monodisperse single core QDs and $38 \mathrm{~nm}$ for the QD clusters (Fig. 3(c)). The fluorescence intensities of the single core QDs and clustered QDs are compared in Fig. 3(d). With excitation at $450 \mathrm{~nm}$ and the same optical density, the fluorescence intensity from the QD clusters was 1.6 times stronger than that of single core QDs, offering potential improvements in ultrasensitive detection and imaging of cells with the clustered QDs [34]. It was noticed that there was a slight blue shift for the single core nanoparticles, possibly caused by erosion of the QDs in water [20]. However, this phenomenon needs to be further investigated.

Using the PEO- $b-\mathrm{P} \gamma$ MPS copolymer and the coating strategy reported here, multifunctional magnetic fluorescent IONP-QD hybrid nanoparticles were also obtained. The TEM image in Fig. 4(a) reveals that the IONP-QD hybrid nanoparticles are composed of a larger IONP and a smaller QD. This is consistent with measurements by DLS, which showed that the hybrid nanoparticles have a hydrodynamic size of $26 \mathrm{~nm}$ (Fig. 4(b)). Since single core IONPs have a

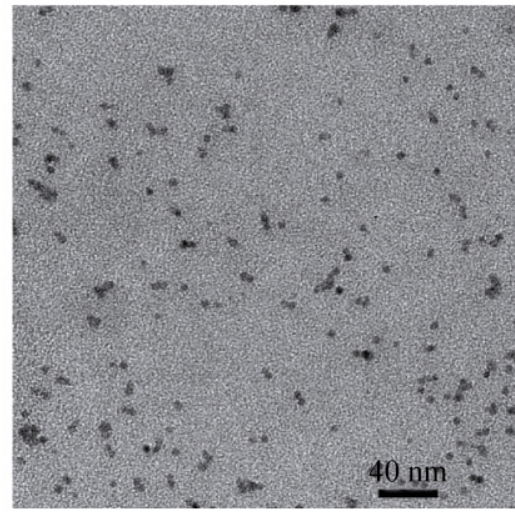

(a)

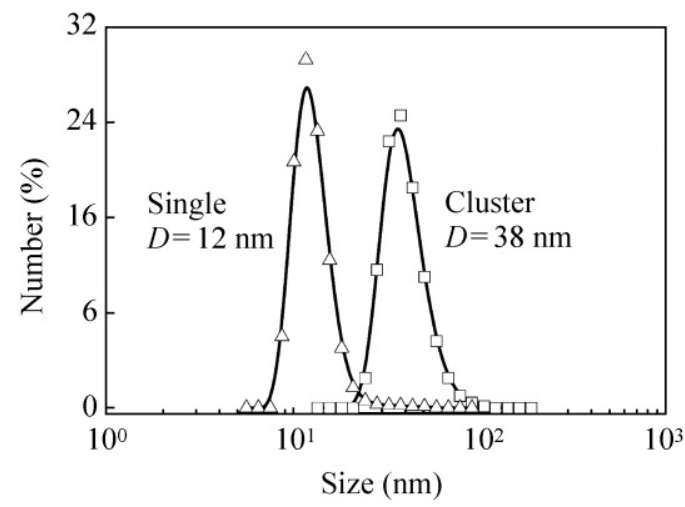

(c)

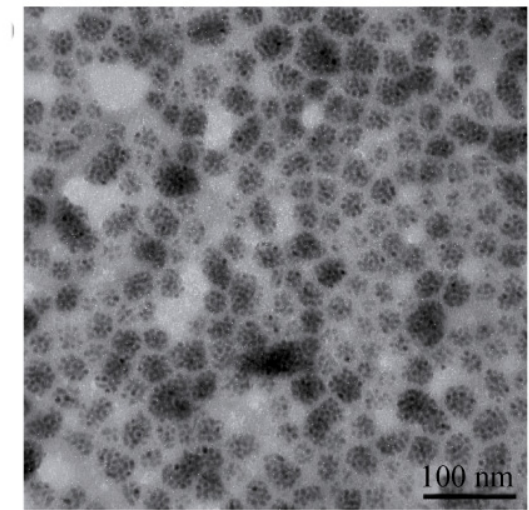

(b)

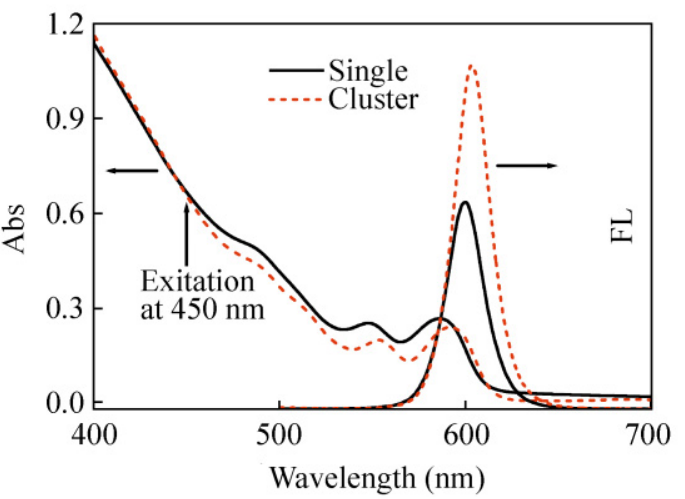

(d)

Figure 3 TEM images of PEO- $b$-P $\gamma$ MPS-coated monodisperse single core (a) and clustered (b) QDs. The hydrodynamic size distributions of monodisperse single core and clustered QDs in water were obtained from DLS (c). Optical absorption and fluorescence spectra of monodisperse single core (solid line) and clustered (dashed line) QDs in water show a higher intensity from the clustered QDs than from single core QDs (d) 
hydrodynamic size of $23 \mathrm{~nm}$, this suggests that each hybrid nanoparticle has a single IONP core $(13 \mathrm{~nm})$. From the ratio of IONP and QD used for the preparation of the hybrid nanoparticles and the survey of visible hybrid nanoparticles in the TEM images, it is appears that most of the hybrids consist of one IONP and one QD. Accurate quantification of the ratio of IONPs to QDs in the hybrids and development of a strategy for controlling the ratio of IONPs and QDs in the hybrids are currently underway. Figure 4(c) shows the absorption spectrum of the IONP-QD hybrid nanoparticles. The absorption peak around $560 \mathrm{~nm}$ is attributed to the presence of QDs in the IONP-QD hybrid, which is further confirmed by the absorption and fluorescence emission spectrum after subtracting the background due to IONP (Fig. 4(d)). However, the quantum yield (QY) of the

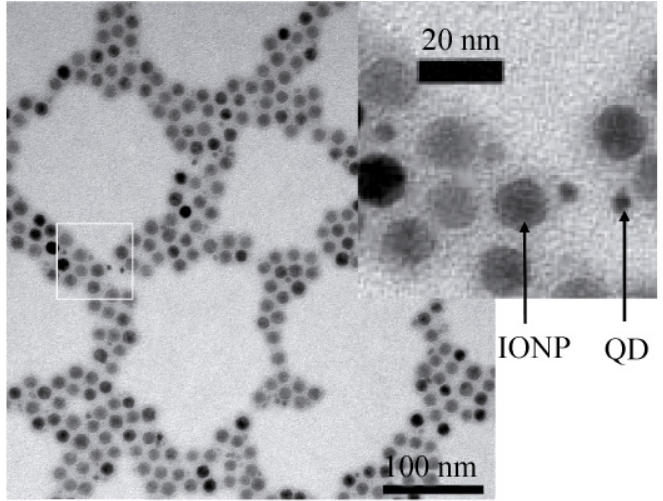

(a)

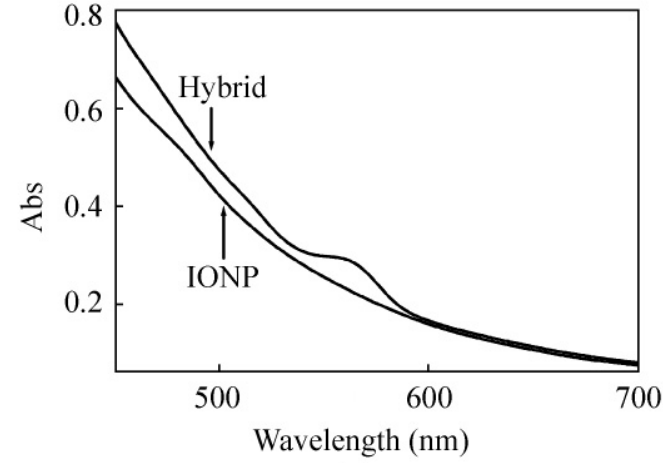

(c)
IONP-QD hybrid nanoparticles was only $17.3 \%$ of the QY of the QDs in the absence of IONPs; this is likely due to the "quenching" effect of the iron oxide. To further confirm the formation of IONP-QD hybrid nanoparticles and investigate the possibility of magnetic separation of the hybrid nanoparticles, an external magnet was applied to the solution of IONP-QD hybrid nanoparticles overnight. Under the force of the magnetic field, the hybrid nanoparticles were accumulated at the bottom of the vial as shown in the insert (A) of Fig. 4(d). To verify the concurrent presence of QDs in the clusters, the sample was excited with a UV lamp, resulting in the observation of fluorescence from the accumulated IONP-QD hybrid nanoparticles at the bottom of the vial as shown in the insert (B) of Fig. 4(d). With gentle shaking, the accumulated IONP-QD hybrid nanoparticles

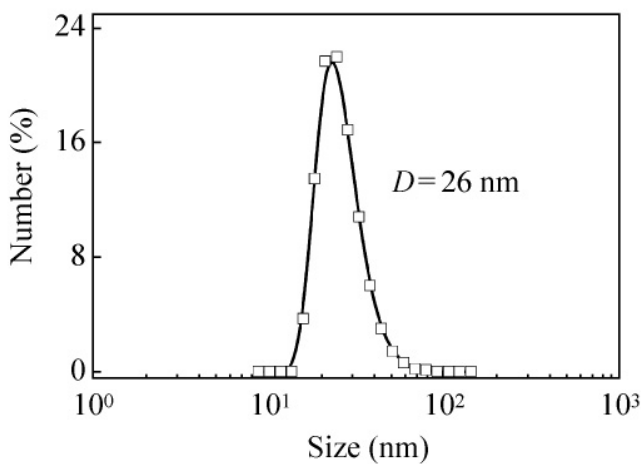

(b)

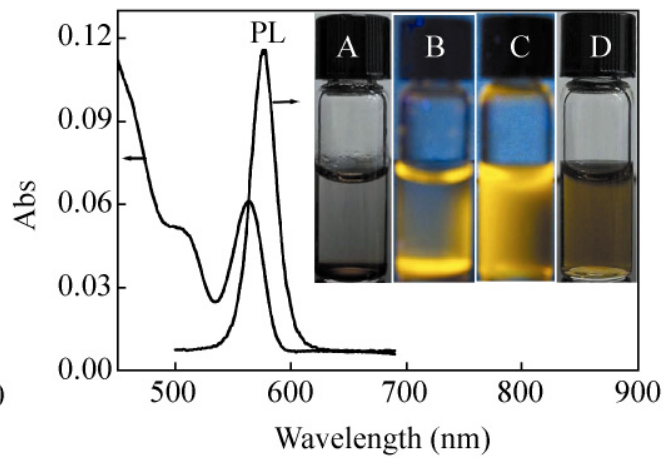

(d)

Figure 4 (a) TEM image of PEO- $b$-P $\gamma$ MPS-coated hybrid nanoparticles. (b) The hydrodynamic size distribution of hybrid IONP-QD nanoparticles in water. (c) Absorption spectra of IONP-QD hybrid nanoparticles and IONPs in water showing the presence of the QD absorption peak. (d) Optical absorption of hybrid IONP-QD nanoparticles with subtracted background of IONPs and fluorescence spectrum of hybrid IONP-QD nanoparticles in water. The insert shows: photographs of the PEO- $b$-P $\gamma$ MPS-coated hybrid magnetic fluorescent nanoparticles after concentrating with a magnet overnight under normal light (vial A) and under a handheld UV lamp (vial B); photographs under UV (vial C) and normal light (vial D) after the vial B was shaken 
were easily redispersed to their original homogeneous state in solution as seen under irradiation with a UV lamp (C) and normal lighting (D). Different from the sequential growth of QDs onto magnetic nanoseeds to afford hybrid nanoclusters, as reported by others [19], we have demonstrated that the PEO- $b$-P $\gamma \mathrm{MPS}$ polymer is capable of forming magnetic fluorescent hybrid nanoparticles with the QD assembled on a single IONP in a simultaneous encapsulation process.

Recently multi-modal imaging probes that can expand the capabilities and functionalities of single nanoparticles have attracted increasing interest for various biomedical applications [40-44]. To demonstrate the potential applications of our magnetic- optical hybrid nanoparticles for cancer cell targeting and imaging, the IONP-QD hybrid nanoparticles coated with functionalized PEO- $b$-P $\gamma$ MPS copolymer were conjugated with the thiolated small peptide cyclo(Arg-Gly-Asp-D-Phe-Cys) (RGD-SH) using the same method reported previously to target tumor integrin $\alpha_{\mathrm{v}} \beta_{3}$ [20]. U87MG human glioma cells overexpressing $\alpha_{\mathrm{v}} \beta_{3}$ integrin were treated with the RGD-conjugated IONP-QD hybrid nanoparticles and then examined by immunohistochemical analysis. Substantial positive Prussian blue staining for iron was observed in the U87MG cells, indicating the binding of the $\alpha_{v} \beta_{3}$ integrin-targeted IONP-QD hybrid nanoparticles (Fig. 5(a)). Confocal microscopic imaging

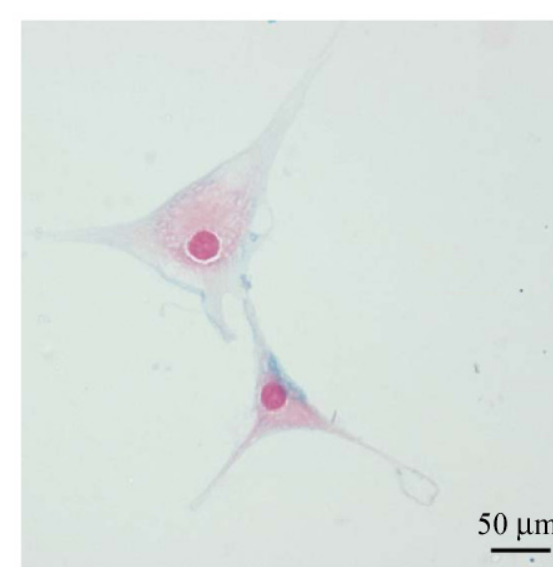

(a)

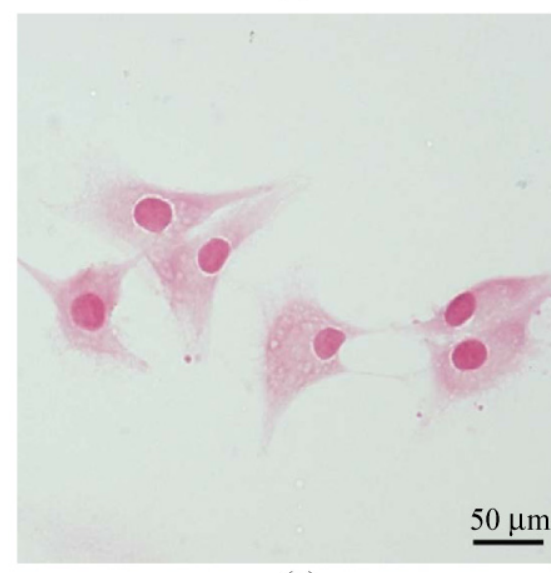

(c)

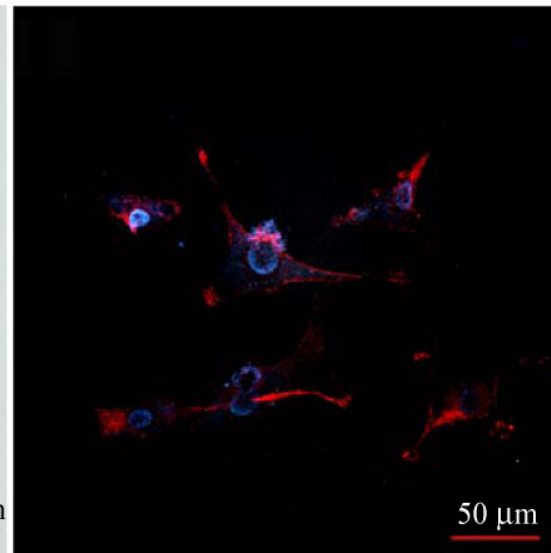

(b)

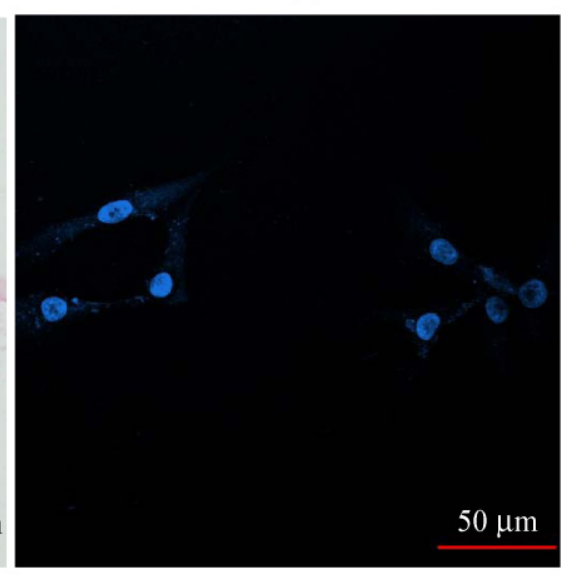

(d)

Figure 5 (a) U87MG human glioma cells treated with RGD-conjugated IONP-QD hybrid nanoparticles showing positive Prussian blue staining for iron from the IONPs. (b) Bright red fluorescence from QDs bound to the cells (blue) is visible in the confocal microscopic image, where the red color instead of yellow around the cell membrane is the result of using a band pass filter (560$615 \mathrm{~nm}$ ). Prussian blue staining for iron (c) and confocal microscopic imaging for detection of QDs (d) in U87MG cells after being incubated with hybrid IONP-QD nanoparticles without RGD. Cells were counterstained with nuclear fast red for Prussian blue staining or DAPI for confocal microscope imaging, respectively 
of those U87MG cells bound with RGD-conjugated hybrid IONP-QD nanoparticles revealed $\alpha_{v} \beta_{3}$ integrintargeted IONP-QD hybrid nanoparticles residing around the cell membrane, as evidenced from the fluorescence from the QDs (Fig. 5(b)). In contrast, after incubation of U87MG cells with hybrid nanoparticles without conjugated RGD ligands, both Prussian blue staining and the confocal microscopic imaging did not show any non-specific binding of IONP-QD hybrid nanoparticles (Figs. 5(c) and 5(d)).

\section{Conclusions}

We report the application of a versatile polysiloxanecontaining block copolymer to prepare and control the formation of monodisperse single core and clustered IONPs and QDs as well as magneticoptical hybrid nanoparticles containing both IONPs and QDs. Clustered IONPs show substantially higher saturation magnetization and relaxivity than their single core nanoparticle counterpart, offering enhanced capabilities in biomedical applications, such as MRI contrast enhancing, magnetic separation, and hyperthermia. Similarly, the clustered QDs exhibit a stronger emission than their single particle counterpart. The hybrid IONP-QDs nanoparticles, after further functionalization with the biomarker-targeting RGD peptide ligand, offer both magnetic and optical properties for potential application as dual-modal imaging nanoprobes and optical imaging-assisted magnetic separations.

\section{Acknowledgements}

The authors wish to thank the staff members of the Robert P. Apkarian Integrated Electron Microscopy Core for their assistance with the electron microscopy work. This work is supported in part by the Emory Molecular Translational Imaging Center with an in vivo Cellular and Molecular Imaging Center grant (ICMIC, No. P50CA128301-01A10003) from the National Cancer Institute (NCI), the Emory-Georgia Tech Nanotechnology Center for Personalized and Predictive Oncology with a grant from the Center of Cancer Nanotechnology Excellence (CCNE, No. U54
CA119338-01) from NCI and a research grant from EmTech Bio, Inc.

Open Access: This article is distributed under the terms of the Creative Commons Attribution Noncommercial License which permits any noncommercial use, distribution, and reproduction in any medium, provided the original author(s) and source are credited.

\section{References}

[1] Kim, J.; Piao, Y.; Hyeon, T. Multifunctional nanostructured materials for multimodal imaging, and simultaneous imaging and therapy. Chem. Soc. Rev. 2009, 38, 372-390.

[2] Laurent, S.; Forge, D.; Port, M.; Roch, A.; Robic, C.; Elst, L. V.; Muller, R. N. Magnetic iron oxide nanoparticles: Synthesis, stabilization, vectorization, physicochemical characterizations, and biological applications. Chem. Rev. 2008, 108, 2064-2110.

[3] Douma, K.; Prinzen, L.; Slaaf, D. W.; Reutelingsperger, C. P. M.; Biessen, E. A. L.; Hackeng, T. M.; Post, M. J.; van Zandvoort, M. A. M. J. Nanoparticles for optical molecular imaging of atherosclerosis. Small 2009, 5, 544-557.

[4] Murray, C. B.; Norris, D. J.; Bawendi, M. G. Synthesis and characterization of nearly monodisperse $\mathrm{Cd} E(E=\mathrm{S}, \mathrm{Se}, \mathrm{Te})$ semiconductor nanocrystallites. J. Am. Chem. Soc. 1993, 115, 8706-8715.

[5] De Palma, R.; Peeters, S.; Van Bael, M. J.; Van den Rul, H.; Bonroy, K.; Laureyn, W.; Mullens, J.; Borghs, G.; Maes, G. Silane ligand exchange to make hydrophobic superparamagnetic nanoparticles water-dispersible. Chem. Mater. 2007, 19, 1821-1831.

[6] Jun, Y. W.; Huh, Y. M.; Choi, J. S.; Lee, J. H.; Song, H. T.; Kim, S.; Yoon, S.; Kim, K. S.; Shin, J. S.; Suh, J. S.; Cheon, J. Nanoscale size effect of magnetic nanocrystals and their utilization for cancer diagnosis via magnetic resonance imaging. J. Am. Chem. Soc. 2005, 127, 5732-5733.

[7] Hezinger, A. F. E.; Tessmar, J.; Gopferich, A. Polymer coating of quantum dots-A powerful tool toward diagnostics and sensorics. Eur. J. Pharm. Biopharm. 2008, 68, 138-152.

[8] Park, J.; Yu, M. K.; Jeong, Y. Y.; Kim, J. W.; Lee, K.; Phan, V. N.; Jon, S. Antibiofouling amphiphilic polymer-coated superparamagnetic iron oxide nanoparticles: Synthesis, characterization, and use in cancer imaging in vivo. J. Mater. Chem. 2009, 19, 6412-6417.

[9] Gao, X. H.; Cui, Y. Y.; Levenson, R. M.; Chung, L. W. K.; Nie, S. M. In vivo cancer targeting and imaging with semiconductor quantum dots. Nat. Biotechnol. 2004, 22, 969-976. 
[10] Pellegrino, T.; Manna, L.; Kudera, S.; Liedl, T.; Koktysh, D.; Rogach, A. L.; Keller, S.; Radler, J.; Natile, G.; Parak, W. J. Hydrophobic nanocrystals coated with an amphiphilic polymer shell: A general route to water soluble nanocrystals. Nano Lett. 2004, 4, 703-707.

[11] Lee, H.; Lee, E.; Kim, D. K.; Jang, N. K.; Jeong, Y. Y.; Jon, S. Antibiofouling polymer-coated superparamagnetic iron oxide nanoparticles as potential magnetic resonance contrast agents for in vivo cancer imaging. J. Am. Chem. Soc. 2006, 128, 7383-7389.

[12] Ai, H.; Flask, C.; Weinberg, B.; Shuai, X.; Pagel, M. D.; Farrell, D.; Duerk, J.; Gao, J. M. Magnetite-loaded polymeric micelles as ultrasensitive magnetic-resonance probes. $A d v$. Mater. 2005, 17, 1949-1952.

[13] Nasongkla, N.; Bey, E.; Ren, J. M.; Ai, H.; Khemtong, C.; Guthi, J. S.; Chin, S. F.; Sherry, A. D.; Boothman, D. A.; Gao, J. M. Multifunctional polymeric micelles as cancer-targeted, MRI-ultrasensitive drug delivery systems. Nano Lett. 2006, 6, 2427-2430.

[14] Kim, B. S.; Taton, T. A. Multicomponent nanoparticles via self-assembly with cross-linked block copolymer surfactants. Langmuir 2007, 23, 2198-2202.

[15] Kim, B. S.; Qiu, J. M.; Wang, J. P.; Taton, T. A. Magnetomicelles: Composite nanostructures from magnetic nanoparticles and cross-linked amphiphilic block copolymers. Nano Lett. 2005, 5, 1987-1991.

[16] Euliss, L. E.; Grancharov, S. G.; O'Brien, S.; Deming, T. J.; Stucky, G. D.; Murray, C. B.; Held, G. A. Cooperative assembly of magnetic nanoparticles and block copolypeptides in aqueous media. Nano Lett. 2003, 3, 1489-1493.

[17] Zhang, L.; Lin, J.; Lin, S. Self-assembly behavior of amphiphilic block copolymer/nanoparticle mixture in dilute solution studied by self-consistent-field theory/density functional theory. Macromolecules 2007, 40, 5582-5592.

[18] Neuberger, T.; Schopf, B.; Hofmann, H.; Hofmann, M.; von Rechenberg, B. Superparamagnetic nanoparticles for biomedical applications: Possibilities and limitations of a new drug delivery system. J. Magn. Magn. Mater. 2005, 293, 483-496.

[19] Gao, J. H.; Zhang, B.; Gao, Y.; Pan, Y.; Zhang, X. X.; Xu, B. Fluorescent magnetic nanocrystals by sequential addition of reagents in a one-pot reaction: A simple preparation for multifunctional nanostructures. J. Am. Chem. Soc. 2007, 129, 11928-11935.

[20] Chen, H. W.; Wang, L. Y.; Yeh, J.; Wu, X. Y.; Cao, Z. H.; Wang, Y. A.; Zhang, M. M.; Yang, L.; Mao, H. Reducing nonspecific binding and uptake of nanoparticles and improving cell targeting with an antifouling PEO- $b$-P $\gamma$ MPS copolymer coating. Biomaterials 2010, 31, 5397-5407.
[21] Yu, W. W.; Falkner, J. C.; Yavuz, C. T.; Colvin, V. L. Synthesis of monodisperse iron oxide nanocrystals by thermal decomposition of iron carboxylate salts. Chem. Commun. 2004, 2306-2307.

[22] Li, J. J.; Wang, Y. A.; Guo, W. Z.; Keay, J. C.; Mishima, T. D.; Johnson, M. B.; Peng, X. G. Large-scale synthesis of nearly monodisperse $\mathrm{CdSe} / \mathrm{CdS}$ core/shell nanocrystals using air-stable reagents via successive ion layer adsorption and reaction. J. Am. Chem. Soc. 2003, 125, 12567-12575.

[23] Chen, H. W.; Wu, X. Y.; Duan, H. W.; Wang, Y. A.; Wang, L. Y.; Zhang, M. M.; Mao, H. Biocompatible polysiloxanecontaining diblock copolymer PEO- $b$-P $\gamma$ MPS for coating magnetic nanoparticles. ACS Appl. Mater. Interf. 2009, 1, 2134-2140.

[24] Atkins, R. C. Colorimetric determination of iron in vitamin supplement tablets. A general chemistry experiment. J. Chem. Educ. 1975, 52, 550-550.

[25] Lalatonne, Y.; Richardi, J.; Pileni, M. P. Van der Waals versus dipolar forces controlling mesoscopic organizations of magnetic nanocrystals. Nat. Mater. 2004, 3, 121-125.

[26] Luttinger, J. M.; Tisza, L. Theory of dipole interaction in crystals. Phys. Rev. 1946, 70, 954-964.

[27] Seo, S. B.; Yang, J.; Lee, T. I.; Chung, C. H.; Song, Y. J.; Suh, J. S.; Yoon, H. G.; Huh, Y. M.; Haam, S. Enhancement of magnetic resonance contrast effect using ionic magnetic clusters. J. Colloid Interf. Sci. 2008, 319, 429-434.

[28] Berret, J. F.; Schonbeck, N.; Gazeau, F.; El Kharrat, D.; Sandre, O.; Vacher, A.; Airiau, M. Controlled clustering of superparamagnetic nanoparticles using block copolymers: Design of new contrast agents for magnetic resonance imaging. J. Am. Chem. Soc. 2006, 128, 1755-1761.

[29] Matsumoto, Y.; Jasanoff, A. $T_{2}$ relaxation induced by clusters of superparamagnetic nanoparticles: Monte Carlo simulations. Magn. Reson. Imaging 2008, 26, 994-998.

[30] Larsen, B. A.; Haag, M. A.; Serkova, N. J.; Shroyer, K. R.; Stoldt, C. R. Controlled aggregation of superparamagnetic iron oxide nanoparticles for the development of molecular magnetic resonance imaging probes. Nanotechnology 2008, 19, 265102.

[31] Duan, H. W.; Kuang, M.; Wang, X. X.; Wang, Y. A.; Mao, H.; Nie, S. M. Reexamining the effects of particle size and surface chemistry on the magnetic properties of iron oxide nanocrystals: New insights into spin disorder and proton relaxivity. J. Phys. Chem. C 2008, 112, 8127-8131.

[32] Lee, J. H.; Jun, Y. W.; Yeon, S. I.; Shin, J. S.; Cheon, J. Dual-mode nanoparticle probes for high-performance magnetic resonance and fluorescence imaging of neuroblastoma. Angew. Chem.Int. Ed. 2006, 45, 8160-8162. 
[33] Barick, K. C.; Aslam, M.; Lin, Y. P.; Bahadur, D.; Prasad, P. V.; Dravid, V. P. Novel and efficient MR active aqueous colloidal $\mathrm{Fe}_{3} \mathrm{O}_{4}$ nanoassemblies. J. Mater. Chem. 2009, 19, 7023-7029.

[34] Yang, J.; Dave, S. R.; Gao, X. H. Quantum dot nanobarcodes: Epitaxial assembly of nanoparticle-polymer complexes in homogeneous solution. J. Am. Chem. Soc. 2008, 130, 52865292.

[35] Dagata, J. A.; Farkas, N.; Dennis, C. L.; Shull, R. D.; Hackley, V. A.; Yang, C.; Pirollo, K. F.; Chang, E. H. Physical characterization methods for iron oxide contrast agents encapsulated within a targeted liposome-based delivery system. Nanotechnology 2008, 19, 305101.

[36] Goya, G. F.; Berquo, T. S.; Fonseca, F. C.; Morales, M. P. Static and dynamic magnetic properties of spherical magnetite nanoparticles. J. Appl. Phys. 2003, 94, 3520-3528.

[37] Han, D. H.; Wang, J. P.; Luo, H. L. Crystallite size effect on saturation magnetization of fine ferrimagnetic particles. J. Magn. Magn. Mater. 1994, 136, 176-182.

[38] Hadjipanayis, C. G.; Bonder, M. J.; Balakrishanan, S.; Wang, X.; Mao, H.; Hadjipanayis, G. C. Metallic iron nanoparticles for MRI contrast enhancement and local hyperthermia. Small 2008, 4, 1925-1929.

[39] Kim, J.; Piao, Y.; Lee, N.; Park, Y. I.; Lee, I. H.; Lee, J. H.;
Paik, S. R.; Hyeon, T. Magnetic nanocomposite spheres decorated with $\mathrm{NiO}$ nanoparticles for a magnetically recyclable protein separation system. Adv. Mater. 2010, 22, 57-60.

[40] Gu, H. W.; Ho, P. L.; Tsang, K. W. T.; Wang, L.; Xu, B. Using biofunctional magnetic nanoparticles to capture vancomycin-resistant enterococci and other Gram-positive bacteria at ultralow concentration. J. Am. Chem. Soc. 2003, 125, 15702-15703.

[41] Yi, D. K.; Selvan, S. T.; Lee, S. S.; Papaefthymiou, G. C.; Kundaliya, D.; Ying, J. Y. Silica-coated nanocomposites of magnetic nanoparticles and quantum dots. J. Am. Chem. Soc. 2005, 127, 4990-4991.

[42] Kim, J.; Lee, J. E.; Lee, J.; Jang, Y.; Kim, S. W.; An, K.; Yu, J. H.; Hyeon, T. Generalized fabrication of multifunctional nanoparticle assemblies on silica spheres. Angew. Chem. Int. Ed. 2006, 45, 4789-4793.

[43] Mulder, W. J. M.; Koole, R.; Brandwijk, R. J.; Storm, G.; Chin, P. T. K.; Strijkers, G. J.; de Mello Donegá, C. M.; Nicolay, K.; Griffioen, A. W. Quantum dots with a paramagnetic coating as a bimodal molecular imaging probe. Nano Lett. 2006, 6, 1-6.

[44] Gao, J. H.; Gu, H. W.; Xu, B. Multifunctional magnetic nanoparticles: Design, synthesis, and biomedical applications. Acc. Chem. Res. 2009, 42, 1097-1107. 